\title{
Asymptomatic severe aortic stenosis: case report and practical application of current ESC and ACC/AHA guidelines
}

\author{
Marko Banovic ${ }^{1,2}$, Bosiljka Vujisic-Tesic ${ }^{1,2}$, Branko Beleslin ${ }^{1,2}$, Milan Nedeljkovic ${ }^{1,2}$ \\ ${ }^{1}$ Cardiology Clinic, Clinical Center of Serbia, ${ }^{2}$ Belgrade Medical School
}

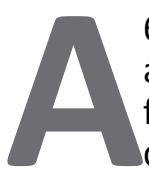

65-year old male with a history of degenerative aortic stenosis (AS) presented to our department for regular echocardiographic evaluation. He denied any symptoms, but he also emphasized that he is not physically active. The clinical exam revealed no specific abnormalities beside the existence of systolic mormour and blunted second aortic sound, his blood pressure was normal (with antyhipertensive therapy) and his ECG was without ST-T segment abnormalities. Analyzed laboratory parameters (complete blood count and biochemistry analysis) were within referent values, but he was also taking atorvastatin for lipid control. His Brain natriuretic peptide level was $28 \mathrm{pg} / \mathrm{ml}$. Echocardiogram confirmed the existence of isolated severe AS (figure 1) with echocardiogaphically observed heavily calcified aortic valve (figure 2) and normal left ventricular ejection fraction (LVEF). To evaluate his symptomatic and functional status we have performed cardio-pulmonary and stressechocardiography testing (as a single test) on semisupine ergobicycle, Ramp 15 protocol. Adequate load was achieved $(>80 \%$ of predicted heart rate and 12 minutes of pedalling, and RER has been achieved at cardiopulmonary testing). The stress-echocardiography test revealed

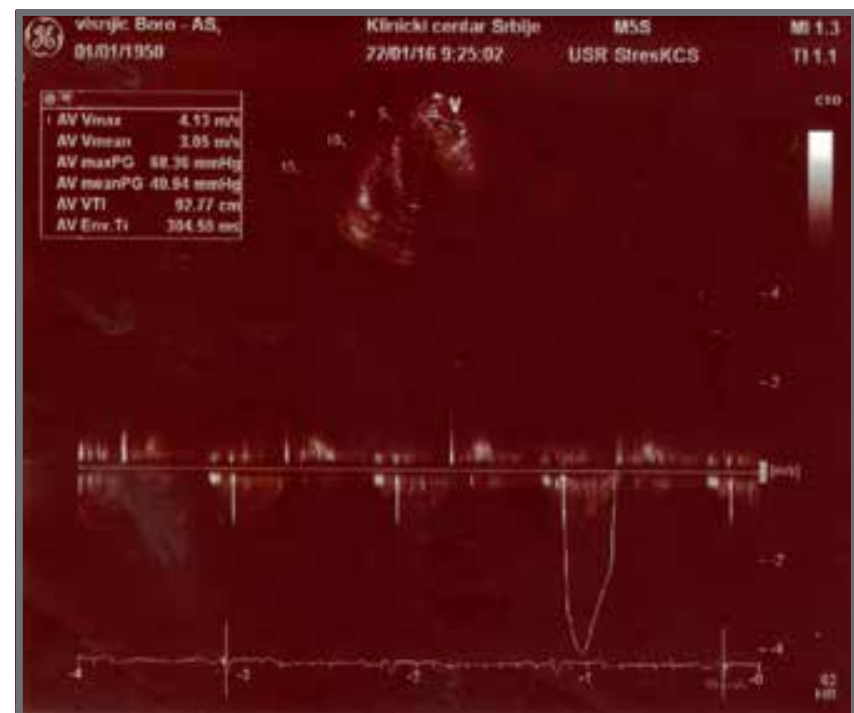

Figure 1. Continues Doppler recording across the aortic valve at rest significant rise in his mean pressure gradient $($ Pmean $=$ $19.6 \mathrm{mmHg}$ ); figure 3 , and normal LV wall motion, while

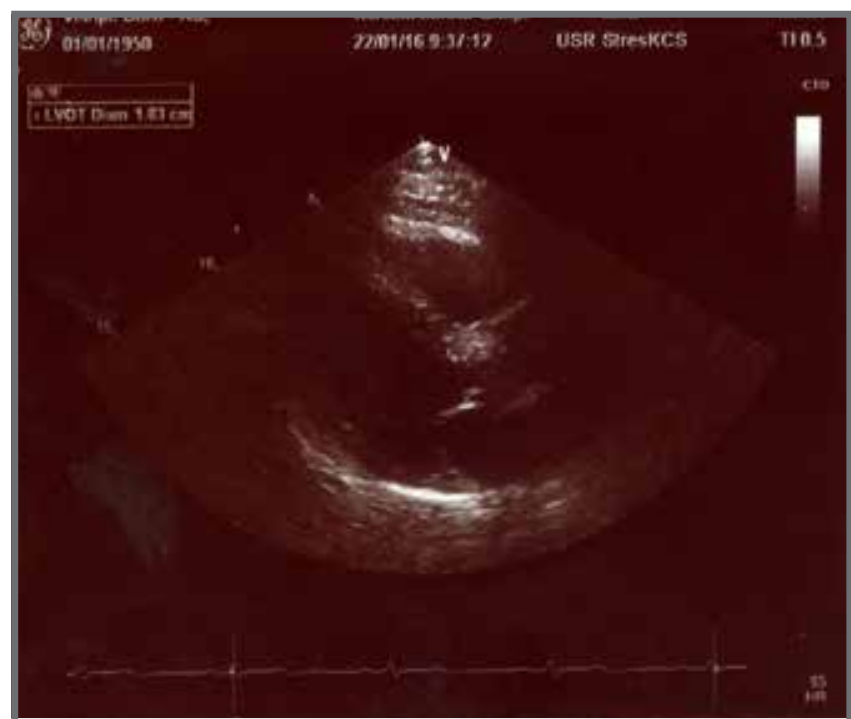

Figure 2. 2-D echocardiographic view of the aortic valve and LVOT

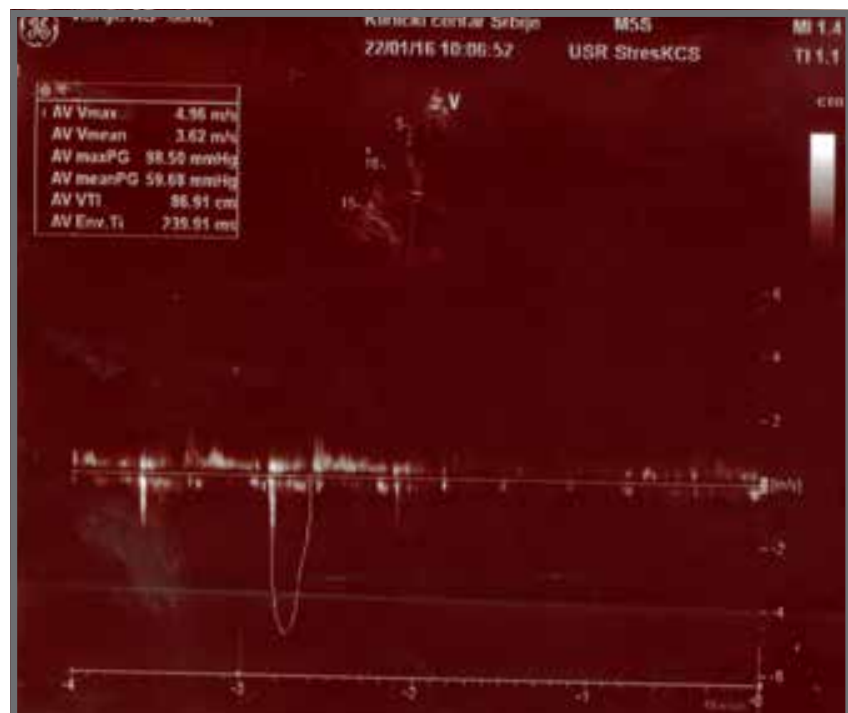

Figure 3. Increase in maximal aortic gradient after exercise testing 
his cardio-pulmonary test revealed preserved cardiac and pulmonary function. The subsequent coronary angiogram revelead no significant coronary artery stenosis. After all the testing and anaysis performed, the remained question was should we reffer this patient to the aortic valve replacement (AVR), or should we opt for "watchful waiting" strategy.

\section{Discussion}

Asymptomatic patients with isolated severe AS and normal LVEF represent one of the most challenging and probably the least explored group of patients in contemporary cardiovascular medicine (stage $\mathrm{C} 1$ in current ACC/AHA valvular guidelines). This additionaly gains importance if one is aware that AS is one of the most common valvular diseases encountered in clinical practice, and affects $\sim 5 \%$ of adults above the age of 65 years $^{1}$. Moreover, its prevalence is projected to increase over the next decade with the aging population ${ }^{2-3}$. Untreated, symptomatic severe AS is associated with a dismal prognosis, with estimated mortality around $2-2.5 \%$ per month ${ }^{4-5}$. In this context the treatment of symptomatic AS patients is clear and includes either surgical AVR or TAVR (transcutaneous aortic valve replacement). Conversely, the treatment of asymptomatic patients with severe AS is unclear and a matter of ongoing debate. The main argument for this ongoing debate is that the risk of sudden death in these patients with severe AS and without symptoms is not $0 \%$, but $\sim 1 \%$ to $1.5 \%$ per year ${ }^{6}$. Importantly, $\sim 70 \%$ of sudden deaths in patients with asymptomatic severe AS are not preceded by any of the classical AS symptoms, thus representing the first clinical manifestation of $\mathrm{AS}^{5-7}$.

Given the current low periprocedural mortality rate for isolated AVR, earlier intervention has been increasingly advocated. Although current ACC/AHA and European guidelines recommend AVR for selected patients with asymptomatic severe AS, in practice, a "watchful waiting" strategy applies for the vast majority of asymptomatic patients, with intervention scheduled once symptoms emerge or left LV systolic dysfunction develops ${ }^{8-9}$. In that sense, of particular importance are two facts:

a) no randomized trial in the setting of asymptomatic severe AS has ever been conducted (one has recently started; ${ }^{10-11}$

b) current ESC and ACC/AHA guidelines are not fully concordant regarding referral to AVR in certain subgroups of asymptomatic severe AS patients.

It is important to emphasize that the level of evidence substantiating each of these recommendations is either $\mathrm{B}$ or C, meaning that they are made on the basis of small, retrospective, observational studies or expert consensus opinions, with no randomized clinical trial available. The consequence of the lack of the randomized trials and discordance in ESC - ACC/AHA guidelines is that decisions are made on individual basis. For this reason, a patient with identical echocardiographic/clinical characteristics may have surgery in one institution, but not in the neighboring one.

The current ESC guidelines (ESC) state that AVR can be considered in subgroup of asymptomatic patients with isolated severe AS in whom $P_{\text {mean }}$ rises $>18 \mathrm{mmHg}$ during exercise testing (the case of our patient), or in patients with elevated BNP and/or in case of severe LV hypertrophy (Class IIB, level of evidence C). This ESC recommendation is based on two prospective, single-center studies in which increase in $\mathrm{P}_{\text {mea }} \mathrm{n}>18 \mathrm{mmHg}$ and $>$ $20 \mathrm{mmHg}$, respectively, was independently associated with mid - and long term adverse events ${ }^{12-13}$. Conversely, current ACC/AHA guidelines are not even considering these subgroups of patients for AVR. Our patient belongs exactly to this subgroup of patients, who are differently recognized and stratified (or unrecognized) in ESC and ACC/AHA guidelines.

After analyzing all data described above, and talking to patient, we opted to recommend to patient AVR. The surgery was successfully performed and mechanical St Jude valve was implanted. Now, 3.5 months after the surgery patient is feeling well and no complication has been observed. In the absence of clear guidelines how to manage this patient we opted for surgery for several reasons:

1) detecting symptom onset in this patient might be notoriously difficult, particularly considering his sedentary way of life

2) AS progression could be highly variable and unpredictable, and rapid deterioration may occur

3) operative risk would only increase with patient age. For example, if no comorbidities develop during 2 years of follow-up, the Society of Thoracic Surgeons risk calculator for cardiac surgery projects that as age increases from 73 to 75 years, absolute operative mortality risk increases by $0.1 \%$ and combined mortality and serious morbidity increases by $0.5 \%{ }^{14}$. Obviously, new significant disease would only make the operative risk even higher.

An important addition to our analysis and decision making was emerging evidence of elective AVR usefulness. Four observational studies, which in total included 2.486 patients, compared outcomes of patients with asymptomatic severe AS undergoing early AVR to those treated with medical therapy only $(5-7,15)$. Five hundred twenty-two (21\%) patients underwent early AVR, and 1,964 (79\%) patients underwent a conservative approach. The very recent polled analysis of these studies by Genereux et al indicated that patients with severe asymptomatic AS have 3.5-fold higher rate of all-cause death with a watchful-waiting strategy compared with AVR. Although these studies have limitations and were probably biased in several ways (i.e no stress test were performed to confirm asymptomatic status; also is possible that some of the patients were not offered AVR in the first place because of their increased operative risk) they are indicative for beneficial effect of early/elective AVR. This might be especially true in the era of low periprocedural mortality rates for isolated AVR, reported in high-volume centers ${ }^{16}$. However, despite of the presented case who was successfully electively operated, most of the asymptomatic patients with isolated severe AS and normal LVEF, on the basis of the current evidence, should be managed conservatively, with close monitoring to detect new onset of symptoms, increasing AS se- 
verity, deterioration in LV function, or other risk factors that might prompt consideration of early AVR.

\section{Conclusion}

The optimal approach to the individual asymptomatic patient with severe isolated AS and with normal LVEF is best made by an expert heart team consisting of cardiologists, interventional cardiologists, cardiac surgeons, imaging specialists, and nurses. Given the uncertainty regarding the value of AVR in asymptomatic severe $A S$ and the large and increasing number of affected patients, a randomized clinical trial comparing AVR to conservative treatment is warranted.

\section{References:}

1. Natural history, diagnostic approaches, and therapeutic strategies for patients with asymptomatic severe aortic stenosis. J Am Coll Cardiol 2016; in press. http://dx.doi.org/10.1016/j.jacc.2016.0 2.057

2. Nkomo VT, Gardin JM, Skelton TN, et al. Burden of valvular heart diseases: a population-based study. Lancet 2006; 368:1005-11.

3. Otto CM, Prendergast B. Aortic-valve stenosis - from patients at risk to severe valve obstruction. N Engl J Med 2014; 371:744-56.

4. Bach DS, Siao D, Girard SE, et al. Evaluation of patients with severe symptomatic aortic stenosis who do not undergo aortic valve replacement the potential role of subjectively overestimated operative risk. Circ Cardiovasc Qual Outcomes 2009; 2:533-9.

5. Pellikka PA, Nishimura RA, Bailey KR, et al. The natural history of adults with asymptomatic, hemodynamically significant aortic stenosis. J Am Coll Cardiol 1990;15:1012-7. 2015; 66(25):2827-38.

6. Taniguchi T, Morimoto T, Shiomi H, Ando K, et al. Initial Surgical Versus Conservative Strategies in Patients With Asymptomatic Severe Aortic Stenosis. CURRENT AS Registry Investigators. J Am Coll Cardiol
7. Kang DH, Park SJ, Rim JH, et al. Early surgery versus conventional treatment in asymptomatic very severe aortic stenosis. Circulation 2010; 121:1502-9.

8. Vahanian A, Alfieri O, Andreotti F, et al. Guidelines on the management of valvular heart disease, Eur Heart J 2012; 33(19):2451-2496.

9. Nishimura RA, Otto CM, Bonow RO, et al. 2014 AHA/ACC guideline for the management of patients with valvular heart disease: executive summary: a report of the American College of Cardiology/American Heart Association Task Force on Practice Guidelines. J Am Coll Cardiol 2014;63: 2438-88.

10. Banovic $M$, lung $B, B a r t u n e k J$, et al. Rationale and design of the Aortic Valve replAcemenT versus conservative treatment in Asymptomatic seve Re aortic stenosis (AVATAR trial): A randomized multicenter controlled event-driven trial. Am Heart J 2016; 174:147-153.

11. Banovic M, Nikolic S, Putnik S. A Randomized Trial in Patients With Asymptomatic Severe Aortic Stenosis: A Future Has Begun! J Am Coll Cardiol 2016; 67(16):1970-1.

12. Maréchaux $S$, Hachicha Z, Bellouin $A$, et al. Usefulness of exercise-stress echocardiography for risk stratification of true asymptomatic patients with aortic valve stenosis. Eur Heart J 2010; 31:1390-7.

13. Lancellotti $P$, Lebois $F$, Simon $M$, et al. Prognostic importance of quantitative exercise Doppler echocardiography in asymptomatic valvular aortic stenosis. Circulation 2005; 112:1377-82.

14. Dewey TM, Brown D, Ryan WH, Herbert MA, Prince SL, Mack MJ. Reliability of risk algorithms in predicting early and late operative outcomes in high-risk patients undergoing aortic valve replacement. J Thorac Cardiovasc Surg. 2008; 135:180-187.

15. Pai RG, Kapoor N, Bansal RC, et al. Malignant natural history of asymptomatic severe aortic stenosis: benefit of aortic valve replacement. Ann Thorac Surg 2006; 82:2116-22.

16. Malaisrie SC, McCarthy PM, McGee EC, et al. Contemporary perioperative results of isolated aortic valve replacement for aortic stenosis. Ann Thorac Surg. 2010; 89(3):751-6. 